Article

\title{
Development of Lipomer Nanoparticles for the Enhancement of Drug Release, Anti-Microbial Activity and Bioavailability of Delafloxacin
}

\author{
Md. Khalid Anwer ${ }^{1}$, Muzaffar Iqbal ${ }^{2,3, *}$, Magdy M. Muharram ${ }^{1,4}$, Muqtader Mohammad ${ }^{1}$, \\ Essam Ezzeldin ${ }^{2,3}$, Mohammed F. Aldawsari ${ }^{1}$ (D) Ahmed Alalaiwe ${ }^{1}$ (D) and Faisal Imam ${ }^{5}$ \\ 1 Department of Pharmaceutics, College of Pharmacy, Prince Sattam Bin Abdulaziz University, Al-kharj 11942, \\ Saudi Arabia; m.anwer@psau.edu.sa (M.K.A.); m.moharm@psau.edu.sa (M.M.M.); \\ mo.ahmed@psau.edu.sa (M.M.); moh.aldawsari@psau.edu.sa (M.F.A.); a.alalaiwe@psau.edu.sa (A.A.) \\ 2 Department of Pharmaceutical Chemistry, College of Pharmacy, King Saud University, PO Box 2457, \\ Riyadh 11451, Saudi Arabia; esali@ksu.edu.sa \\ 3 Bioavailability Unit, Central Laboratory, College of Pharmacy, King Saud University, PO Box 2457, \\ Riyadh 11451, Saudi Arabia \\ 4 Department of Microbiology, College of Science, Al-Azhar University, Nasr City, Cairo 11884, Egypt \\ 5 Department of Pharmacology and Toxicology, College of Pharmacy, King Saud University, Riyadh 11451, \\ Saudi Arabia; fimam@ksu.edu.sa \\ * Correspondence: muziqbal@ksu.edu.sa; Tel.: +966-14697565; Fax: +966-14676220
}

Received: 5 February 2020; Accepted: 6 March 2020; Published: 11 March 2020

\begin{abstract}
Delafloxacin (DFL) is a novel potent and broad-spectrum fluoroquinolone group of antibiotics effective against both Gram-positive and negative aerobic and anaerobic bacteria. In this study, DFL-loaded stearic acid (lipid) chitosan (polymer) hybrid nanoparticles (L-P-NPs) have been developed by single-emulsion-solvent evaporation technique. The mean particle size and polydispersity index (PDI) of optimized DFL-loaded L-P-NPs (F1-F3) were measured in the range of 299-368 nm and 0.215-0.269, respectively. The drug encapsulation efficiency (EE\%) and loading capacity (LC\%) of DFL-loaded L-P-NPs (F1-F3) were measured in the range of $64.9-80.4 \%$ and $1.7-3.8 \%$, respectively. A sustained release of DFL was observed from optimized DFL-loaded L-P-NPs (F3). Minimum inhibitory concentration (MIC) values of the DFL-loaded L-P-NPs (F3) appeared typically to be four-fold lower than those of delafloxacin in the case of Gram-positive strains and was 2-4-fold more potent than those of delafloxacin against Gram-negative strains. The pharmacokinetic study in rats confirmed that the bioavailability (both rate and extent of absorption) of DFL-loaded L-P-NPs was significantly higher (2.3-fold) than the delafloxacin normal suspension. These results concluded that the newly optimized DFL-loaded L-P-NPs were more potent against both Gram-positive and negative strains of bacteria and highly bioavailable in comparison to delafloxacin normal suspension.
\end{abstract}

Keywords: delafloxacin; LIPOMER; drug release; antimicrobial activity; pharmacokinetic

\section{Introduction}

Acute bacterial skin and skin structure infections (ABSSSIs) and community-acquired pneumonia (CAP) are considered as the most common type of infection which require hospitalization, and are the leading cause of mortality and morbidity worldwide [1-3]. Delafloxacin (DFL) is a novel fourth-generation fluoroquinolone group of antibiotics approved for treatment in adults for ABSSSI and CAP $[4,5]$. Unlike other fluoroquinolones, there is the absence of a basic group next to the fluorinated ring in the chemical structure of DFL. This change results in a weak acid property, and therefore, DFL usually exists in a deprotonated form at a neutral $\mathrm{pH}$ with a $\mathrm{pKa}$ value of 5.4, providing enhanced 
antibacterial potency in an acidic environment and reducing the minimum inhibitory concentrations (MICs). These properties also lead to increased accumulation and better distribution characteristics into infected target tissues than other marketed fluoroquinolones [6-8]. Moreover, the enzyme-inhibiting effects of DFL against DNA gyrase and topoisomerase IV is more balanced in comparison to other fluoroquinolones. Therefore, DFL exhibits very low frequencies of spontaneous mutation in vitro as an equipotent enzyme inhibition limit resistance $[9,10]$. DFL possesses potent and broad-spectrum antibiotic activities against both Gram-positive and negative aerobic and anaerobic bacteria, including bactericidal effects against methicillin-resistant Staphylococcus aureus (MRSA) [6].

DFL shows rapid absorption after oral administration and peak plasma concentration $\left(\mathrm{C}_{\max }\right)$, usually achieved within $1-2.5 \mathrm{~h}$ in healthy volunteers. It is widely distributed to body fluids with the volume of distribution at a steady state $\left(\mathrm{V}_{\mathrm{SS}}\right)$ in the range of 34-41 L and high plasma protein binding of $83-84 \%$ [11]. In compared with other flouroquinolones, DFL has low absolute bioavailability (58.8\%), which may be due to its poor solubility $(\approx 0.06 \mathrm{mg} / \mathrm{mL}$ in water). Therefore, the approved IV formulation of DFL contains sulfobutylether- $\beta$-cyclodextrin (SBECD) to enhance its solubility and stability [12]. The recommended oral dose of DFL is $450 \mathrm{mg}$, which is administered every $12 \mathrm{~h}$ daily. Frequent administration of conventional DFL formulation might be needed due to its low bioavailability and rapid metabolism with a mean half-life of $\leq 2.5 \mathrm{~h}$ after administration [13-15]. However, frequent administration of antibiotics is usually discouraging for patient compliance reasons, and it may lead to resistance, especially for CA-RTIs treatment [16].

Liposomes are believed to be an excellent biocompatible vesicular system with similarities to the biological membrane. The main concern to vesicular systems is stability, low drug encapsulation and burst drug release. However, polymer-based nanoparticles exhibit better stability over liposomes and also show sustained drug release for extended periods of time. Polymeric nanoparticles are synthesized by using synthetic (e.g., PLGA) and natural (e.g., chitosan) polymers. The use of organic solvents during the synthesis of polymeric nanoparticles limits their application [17-19]. The limitations of both liposomes and polymeric nanoparticles can be overcome by the synthesis of lipid polymer hybrid nanoparticles (L-P-NPs), which possess both lipid and polymeric carriers. L-P-NPs have received increasing interest in recent years, due to their superior characteristics and advantages over biopolymer-based colloidal nanoparticles [20,21]. L-P-NPs are effective in encapsulating the hydrophobic molecules with a higher drug payload than biopolymer-based nanoparticles due to their nano-range size and large surface areas. In addition, they improve drug stability and have the ability to improve the oral bioavailability of poorly water-soluble drugs [22]. Chitosan is a natural cationic polysaccharide obtained by chitin deacetylation. Due to its unique characteristics, such as nontoxicity, biocompatibility and biodegradability, as well as its favorable muco-adhesiveness and bio-membrane permeability, chitosan-coated solid-lipid nanoparticles have also been recently reported to effectively promote the in vivo absorption of poorly soluble drugs $[19,23]$. Recently, lipid-chitosan hybrid nanoparticles (LIPOMER nanoparticles) have been used for the oral delivery of some poorly soluble drugs $[19,24,25]$. In addition, the role of lipid-polymer hybrid nanoparticles in encapsulating antibiotics, e.g., chitosan-coated lipid nanoparticles loaded with rifampicin, have been evaluated for the better management of tuberculosis [26,27]. Therefore, the aim of this study was the development and optimization of novel DFL-loaded stearic acid (lipid) chitosan (polymer) hybrid nanoparticles (L-P-NPs) and their in-vitro/in vivo characterizations.

\section{Materials and Methods}

\subsection{Chemicals and Reagents}

DFL was purchased from Beijing Mesochem Technology (Beijing, China). Stearic acid, chitosan and pluronic 127 were purchased from Sigma Aldrich (St. Louis, MO, USA). All chemicals and solvents used in this study were analytical/HPLC grade. Ultra-pure water was collected from the Milli-Q water purifier unit (Millipore, Darmstadt, Germany) and was used for aqueous solution preparation. 


\subsection{Preparation of Lipid-Polymer Hybrid Nanoparticles}

DFL-loaded L-P-NPs were prepared by single-emulsion-solvent evaporation technique [25]. Briefly, DFL (10 mg) was dissolved in $2 \mathrm{~mL}$ of prepared lipid (stearic acid) solution in ethyl acetate, and this lipid solution was further emulsified with $10 \mathrm{~mL}$ of chitosan solution $(1 \mathrm{mg} / \mathrm{mL})$ in $1 \%$ w/v acetic acid solution containing pluronic 127 surfactant $(50 \mathrm{mg}$ ) under probe sonication (Ultrasonic processor, gx-130, Berlin, Germany) for $3 \mathrm{~min}$ at $60 \%$ voltage efficiency at a temperature of $25^{\circ} \mathrm{C}$ (Table 1$)$. The volatile organic solvent was evaporated by magnetic stirrer at $40{ }^{\circ} \mathrm{C}$ overnight. The DFL-loaded L-P-NPs were separated from the bulk aqueous solution by high-speed centrifugation (12,000 rpm) for $30 \mathrm{~min}$, subsequently washed three times with cold distilled water and, finally, freeze-dried (Millirock Technology, Kingston, NY, USA).

Table 1. Composition of delafloxacin (DFL)-loaded stearic acid (lipid) chitosan (polymer) hybrid nanoparticles (L-P-NPs).

\begin{tabular}{ccccc}
\hline Formulae & DFL $(\mathbf{m g})$ & Stearic Acid $(\mathbf{m g})$ & Chitosan $(\mathbf{m g})$ & Pluronic $\mathbf{1 2 7}(\mathbf{m g})$ \\
\hline F1 & 10 & 100 & 10 & 50 \\
F2 & 10 & 200 & 10 & 50 \\
F3 & 10 & 400 & 10 & 50 \\
\hline
\end{tabular}

\subsection{Particle Characterization of DFL Loaded L-P-NPS}

Prepared L-P-NPs were evaluated for particle size, polydispersity index (PDI) and zeta potential (ZP). The mean size and PDI of L-P-NPs (F1-F3) were measured using Malvern zeta sizer (ZEN-3600, Malvern Instruments Ltd., Holtsville, NY, USA) at $25 \pm 2{ }^{\circ} \mathrm{C}$, and dynamic light scattering (DLS) was set $90^{\circ}$ [28]. The freshly prepared DFL-loaded L-P-NPs dispersion was diluted (100 times) with double-distilled water and sonicated for $10 \mathrm{~min}$. The diluted samples $(3 \mathrm{~mL})$ were taken in disposable plastic cuvette and measured in size and PDI in triplicate. The diluted samples were transferred into glass electrodes in place of a glass cuvette for ZP measurement.

\subsection{Measurement of Drug Encapsulation Efficiency}

An indirect method was followed for the measurement of the percent encapsulation efficiency (EE\%) and drug-loading capacity (LC\%) of DFL-loaded L-P-NPs (F1-F3). Freshly prepared dispersion of nanoparticles was centrifuged (Hermle Labortechnik, Z216MK, Wehingen, Germany) at 15,000 rpm for $10 \mathrm{~min}$ at $-4{ }^{\circ} \mathrm{C}$ to get supernatant. The supernatant was diluted appropriately, and the drug was quantified by UV spectroscopy at $290 \mathrm{~nm}$. The EE\% and DL\% were calculated using the following equations [22]:

$$
\begin{gathered}
\left.\mathrm{EE} \%=\left[\left(\mathrm{D}_{\text {total }}-\mathrm{D}_{\text {free }}\right)\right] / \mathrm{D}_{\text {total }}\right] \times 100 \\
\mathrm{LC} \%=\left[\mathrm{D}_{\text {entrapped }} / \mathrm{W}_{\text {nanoparticle }}\right] \times 100
\end{gathered}
$$

where $\mathrm{D}_{\text {total }}=$ amount of DFL added initially in NPs, $\mathrm{D}_{\text {free }}=\mathrm{DFL}$ in supernatant, $\mathrm{D}_{\text {entrapped }}=\mathrm{DFL}$ entrapped in NPs, and $W_{\text {nanoparticle }}=$ total weight of NPs.

\subsection{Differential Scanning Calorimetry (DSC) Studies}

Thermal behaviors of pure-drug DFL and DFL in L-P-NPs (F1-F3) were studied using a DSC thermal analyzer (Scinco, DSC N-650, Seoul, Korea). Accurately weighed samples were crimped in an aluminum pan by applying pressure and heated over a temperature range of $50-300{ }^{\circ} \mathrm{C}$ at a constant heating rate of $10^{\circ} \mathrm{C} / \mathrm{min}$. The thermal samples were purged with an inert nitrogen gas with a flow rate of $15-20 \mathrm{~mL} / \mathrm{min}$ [23]. 


\subsection{Fourier Transform Infrared Spectroscopy (FTIR)}

FTIR spectra of pure DFL and DFL-loaded L-P-NPs (F1-F3) were recorded using the instrument FTIR spectrometer (Jasco FTIR spectrophotometer, Tokyo, Japan). Each sample was grinded with potassium bromide (10:100), and a transparent film was used by applying pressure. The spectra were recorded in the wavelength range of 4000 to $400 \mathrm{~cm}^{-1}$, and peaks were interpreted with the help of IR software [29].

\subsection{In-Vitro Release Studies}

A comparative release study of pure DFL and optimized DFL-loaded L-P-NPs (F3) was performed using a dialysis bag (Molecular weight cut off, $12 \mathrm{kDa}$ ) [30]. Briefly, pure drug DFL and F3 powder were dispersed in a dialysis bag containing $5 \mathrm{~mL}$ phosphate buffer ( $\mathrm{pH} 7.4$ ), and the bags were further dipped into a beaker containing $25 \mathrm{~mL}$ phosphate buffer at a temperature of $37 \pm 1{ }^{\circ} \mathrm{C}$ with constant shaking at $100 \mathrm{rpm}$ in a biological shaker (LabTech, LBS-030S, Kyonggi, Korea). At predetermined time intervals, $0.5 \mathrm{~mL}$ of samples were withdrawn and analyzed at $290 \mathrm{~nm}$ by UV spectroscopy (Jasco UV spectrophotometer V-630, Tokyo, Japan).

\subsection{Morphology}

The surface morphology and approximate size of the optimized DFL-loaded L-P-NPs (F3) were examined under transmission electron microscopy (TEM; JEOL JEM-1010, Tokyo, Japan). The DFL-loaded L-P-NPs were vortexed for 3 min after dilution with Milli-Q water. A drop of suspended DFL-loaded L-P-NP was pipped out on parafilm, and then the slide of the TEM grid was placed on the drop and left for $15 \mathrm{~min}$. The grid was then removed and placed on tissue paper with the slide for 45 min for drying, then scanned for imaging.

\subsection{In-Vitro Antimicrobial Activity}

\subsubsection{Bacterial Isolates}

For this study, seventeen type strains: Staphylococcus aureus (ATCC 29737), S. aureus (NCTC 6571), Streptococcus pyogenes (ATCC 12344), S. pneumonia (ATCC 10015), S. agalactiae (ATCC12386), Enterococcus faecalis (ATCC 19433), E. faecalis (ATCC 49532), Salmonella typhimurium (ATCC 14028), Pseudomonas aeruginosa (ATCC 10145), Staphylococcus haemolyticus (ATCC 29970), Proteus mirabilis (NCIMB 13283), Enterobacter Aerogenes (ATCC13048), E. aerogenes (NCIMB 10102), Bacillus subtilis (ATCC 11774), Klebsiella pneumonia (ATCC 9633), Bacillus cereus (ATCC 10876) and Staphylococcus epidermidis (ATCC 12228) were examined. In addition, 10 clinical isolates of S. aureus, S. typhimurium, E. coli, S. pneumonia and K. pneumonia were also used. To verify the accuracy of the susceptibility results, strains of Escherichia coli (ATCC 25922), Pseudomonas aeruginosa (ATCC 27853), Staphylococcus aureus (ATCC 29213) and Enterococcus faecalis (ATCC 29212) were used as control organisms.

\subsubsection{Culture Conditions and Media}

Strains were cultured in Mueller Hinton and stored as recommended by the manufacturer. Cultures were incubated for $18 \pm 2 \mathrm{~h}$ at $36 \pm 1{ }^{\circ} \mathrm{C}$ while streptococcal strains were incubated in Mueller-Hinton agar with $5 \%$ sheep blood under microaerobic conditions at $36 \pm 1{ }^{\circ} \mathrm{C}$ for $24 \mathrm{~h}$ in a $\mathrm{CO}_{2}$ incubator.

\subsubsection{McFarland Standard Preparation and Inoculum Size}

McFarland number 0.5 standard was made by mixing of $9.95 \mathrm{~mL}\left(1 \% \mathrm{H}_{2} \mathrm{SO}_{4}\right)$ with $0.05 \mathrm{~mL}$ of $1.175 \%$ barium chloride dihydrate $\left(\mathrm{BaCl}_{2} \cdot 2 \mathrm{H}_{2} \mathrm{O}\right)$ in order to estimate bacterial density [31,32]. Preparation was stored in an airtight bottle and used for comparison of bacterial suspension whenever 
required. Fresh pure cultures were used for the preparation of the inoculum broth dilution of $5 \times 10^{5}$ colony-forming units (CFU) $\mathrm{mL}^{-1}$.

\subsubsection{Minimum Inhibitory Concentration (MIC)}

MICs were assessed by the agar dilution method $[33,34]$ as recommended by the Clinical and Laboratory Standards Institute [35]. MIC values were determined and compared to activities of levofloxacin as a reference standard agent. Following standardized guidelines, bacteria were seeded in a Mueller Hinton agar medium, which was supplemented with different concentrations of DFL and DFL-loaded L-P-NPs (F3). The colony forming unit (CFU) was then counted after $24 \mathrm{~h}$ of incubation.

\subsection{Bio-Analytical Methods}

Our recently published UPLC-MS/MS method [36] was used for the quantitative analysis of DFL in rat plasma samples. All plasma samples were kept frozen at $-80^{\circ}$ before analysis. The method was linear in the concentration range of $3.5-5000 \mathrm{ng} \cdot \mathrm{mL}^{-1}$. The chromatographic separation of DFL and internal standard (rivaroxaban) were performed on UPLC $\mathrm{C}_{18}$ columns using a mobile phase composition of acetonitrile with $0.1 \%$ formic acid and $10 \mathrm{mM}$ ammonium acetate $(60: 40, v / v)$ at a

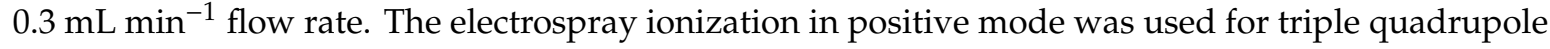
detection. Quantitation was accomplished with multiple reaction monitoring with parent-to-daughter ion transitions of $441.14 \rightarrow 379.09$ for DFL and $436.89 \rightarrow 144.87$ for the internal standard. The sample extractions were performed by the liquid extraction method using ethyl acetate as an extracting agent. The intra and inter-day precision (\% RSD) of the method was $\leq 10.5$, whereas the accuracy was within the range of $-13.2 \%$ to $11.2 \%$ ( $\%$ RE), respectively.

\subsection{Pharmacokinetic Study in Rats}

The comparative bioavailability of newly developed DFL-loaded L-P-NPs (F3) against normal suspension were evaluated in male Wistar albino rats. Twelve animals weighing between 300-350 g were received from the Animal Care Centre, College of Pharmacy, King Saud University, Riyadh, Saudi Arabia. The experimental protocol was approved by the Research Ethics Committee (Reference no. KSU-SE-19-27), and the experiment was carried out according to the guidelines of the Animal Care and Use Committee, King Saud University. Animals were divided into two groups ( $n=6$ each) and were administered DFL normal suspension and DFL-loaded L-P-NPs (F3) after overnight fasting $\left(20 \mathrm{mg} \mathrm{Kg}^{-1}\right)$. Blood samples were withdrawn at different time intervals (pre-dose, 0.5, 1, 1.5, 2, 3, 5, 8, 12 and $24 \mathrm{~h}$ ). After samples were withdrawn, all blood sample were centrifuged at 5000 $\mathrm{g}$ for $10 \mathrm{~min}$, and plasma were separated and stored frozen at $80 \pm 10^{\circ} \mathrm{C}$ until further UPLC-MS/MS analysis.

The pharmacokinetic parameters were calculated using WinNonlin software (Pharsight Co., Mountain View, CA, USA), and all values have been expressed as means \pm standard deviation (SD). The noncompartmental pharmacokinetic model was used to calculate $\mathrm{C}_{\max }$ and the time to reach maximum concentration $\left(\mathrm{T}_{\max }\right)$, $\mathrm{AUC}$ from 0 to $\mathrm{t}\left(\mathrm{AUC}_{0-24}\right)$ and 0 -inf $\left(\mathrm{AUC}_{0-\mathrm{inf}}\right)$, elimination rate constant $(\mathrm{kz})$, half-life $\left(\mathrm{T} \frac{1}{2}\right)$ and mean residence time (MRT).

\section{Results and Discussion}

\subsection{Particle Characterization of DFL-Loaded L-P-NPS}

The mean particle size and PDI of DFL-loaded L-P-NPs (F1-F3) were measured in the range of 299-368 nm and 0.215-0.269, respectively. The results are presented in Table 2. The lowest and largest size of the NPs were measured for F1 $(299 \mathrm{~nm})$ and F3 $(368 \mathrm{~nm})$, respectively. The results suggest that there were larger NPs with an increase in the amount of lipid in the formulation. The PDI values of F1, F2 and F3 was measured as $0.269,0.230$ and 0.215 , respectively. The PDI values less than $\leq 0.3$ are considered monodispersed particles. The $\mathrm{ZP}$ around $\pm 30 \mathrm{mV}$ are considered to be stable colloidal dispersions irrespective of charge (+/-), with the magnitude of charge considered accountable for 
stability. The ZP values of F1, F2 and F3 were found as $+27.8,+20.1$ and $+19.2 \mathrm{mV}$, respectively. The surface charge of the lipid-chitosan hybrid nanoparticles remained positive due to the electrostatic interaction of lipid and chitosan in the formulation. The positive values of $\mathrm{ZP}$ were found due to the dominance of positive charges of chitosans over the negative charges of lipids (stearic acid) [23]. It was observed from the results a slight decrease in ZP with an increase in the amount of lipids (Table 2).

Table 2. Evaluations of DFL-loaded L-P-NPs (mean \pm SD, $n=3$ ). PDI: polydispersity index, ZP: zeta potential, EE\%: drug encapsulation efficiency and LC\%: loading capacity.

\begin{tabular}{ccccccc}
\hline Formulae & Stearic Acid $(\mathbf{m g})$ & Size $(\mathbf{n m})$ & PDI & ZP $(\mathbf{m V})$ & EE\% & LC\% \\
\hline F1 & 100 & $299 \pm 2.7$ & $0.269 \pm 0.042$ & $+27.8 \pm 3.2$ & $64.9 \pm 2.3$ & $3.8 \pm 0.3$ \\
F2 & 200 & $345 \pm 3.9$ & $0.230 \pm 0.018$ & $+20.1 \pm 1.5$ & $76.1 \pm 1.6$ & $2.8 \pm 0.5$ \\
F3 & 400 & $368 \pm 5.2$ & $0.215 \pm 0.015$ & $+19.2 \pm 1.4$ & $80.4 \pm 3.1$ & $1.7 \pm 0.1$ \\
\hline
\end{tabular}

\subsection{Measurement of Drug Encapsulation Efficiency}

The encapsulation efficiencies of DFL-loaded L-P-NPs (F1-F3) were assessed in terms of EE\% and $\mathrm{LC} \%$. The entrapment efficiency gives an idea of the entrapped drug, and the drug loading capacity is about the content of drugs in the nanoparticles. The EE\% and LC\% of DFL in L-P-NPs (F1-F3) were measured in the ranges of $64.9-80.4 \%$ and $1.7-3.8 \%$, respectively (Table 2). It was observed from the results that increases in lipid concentrations lead to an increase in the encapsulation efficiency of DFL, probably due to resistance in the diffusion of the drug. Among the three polymer hybrid lipid NPs, the DFL-loaded L-P-NPs (F3) with compositions of SA (400 mg), CS (10 mg), pluronic 127 (50 mg) and $10 \mathrm{mg}$ of DFL were found optimally with the particle size (368 nm), PDI (0.215), ZP (+19.2 mV), EE\% $(8.4 \%)$ and LC\% (1.7\%). The optimized DFL-loaded L-P-NPs (F3) were further subjected to in vitro release studies, antimicrobial assay and pharmacokinetic studies.

\subsection{DSC Studies}

DSC spectra of pure DFL and DFL in L-P-NPs (F1-F3) were scanned from $50-300^{\circ} \mathrm{C}$ as shown Figure 1. A sharp endothermic peak of pure DFL at $249.32{ }^{\circ} \mathrm{C}$ evidenced its melting temperature, which was found approximately the same as reported in the literature [37]. As we can see from the DSC spectra (Figure 1), the peak of the drug (DFL) completely disappeared in DFL in L-P-NPs (F1-F3). It might be due to the encapsulation of DFL inside the lipid-polymer matrix.

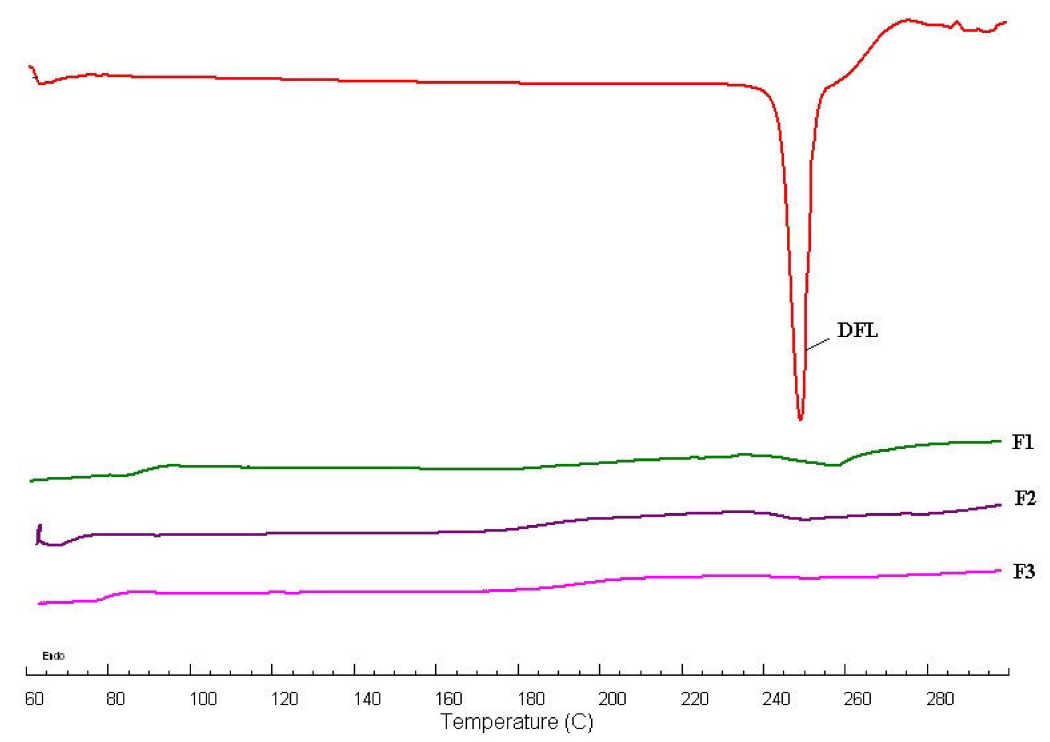

Figure 1. A comparative differential scanning calorimetry (DSC) spectrum of pure delafloxacin (DFL) and DFL-loaded stearic acid (lipid) chitosan (polymer) hybrid nanoparticles (L-P-NPs) (F1-F3). 


\subsection{FTIR Analysis}

The compatibility studies of the polymer (CS), lipid (SA) and drug (DFL) in nanoparticles (F1-F3) were carried out by the FTIR analysis. The comparative FTIR spectra of DFL and DFL-loaded L-P-NPs (F1-F3) were recorded for the identification and characterization of the drug (Figure 2). The major peaks of the initial DFL were assigned corresponding to the functional groups: $-\mathrm{O}-\mathrm{H} \operatorname{str}\left(3345.89 \mathrm{~cm}^{-1}\right)$, $-\mathrm{C}-\mathrm{H} \operatorname{str}\left(3075.94 \mathrm{~cm}^{-1}\right)$, ketone $-\mathrm{C}=\mathrm{O} \operatorname{str}\left(1715.37 \mathrm{~cm}^{-1}\right)$, carboxylic $-\mathrm{C}=\mathrm{O} \operatorname{str}\left(1623.77 \mathrm{~cm}^{-1}\right), \mathrm{C}-\mathrm{F}$ $\operatorname{str}\left(1114.65 \mathrm{~cm}^{-1}\right)$ and $\mathrm{C}-\mathrm{Cl} \operatorname{str}\left(840.63 \mathrm{~cm}^{-1}\right)$ with confirmed drug purity [31], and a reduction in the intensity of FTIR peaks was observed in the absorption spectra of DFL-loaded L-P-NPs (F1-F3) corresponding to the drug in the region of $400-1600 \mathrm{~cm}^{-1}$, which resulted from the overlapping of the CS, SA and DFL.

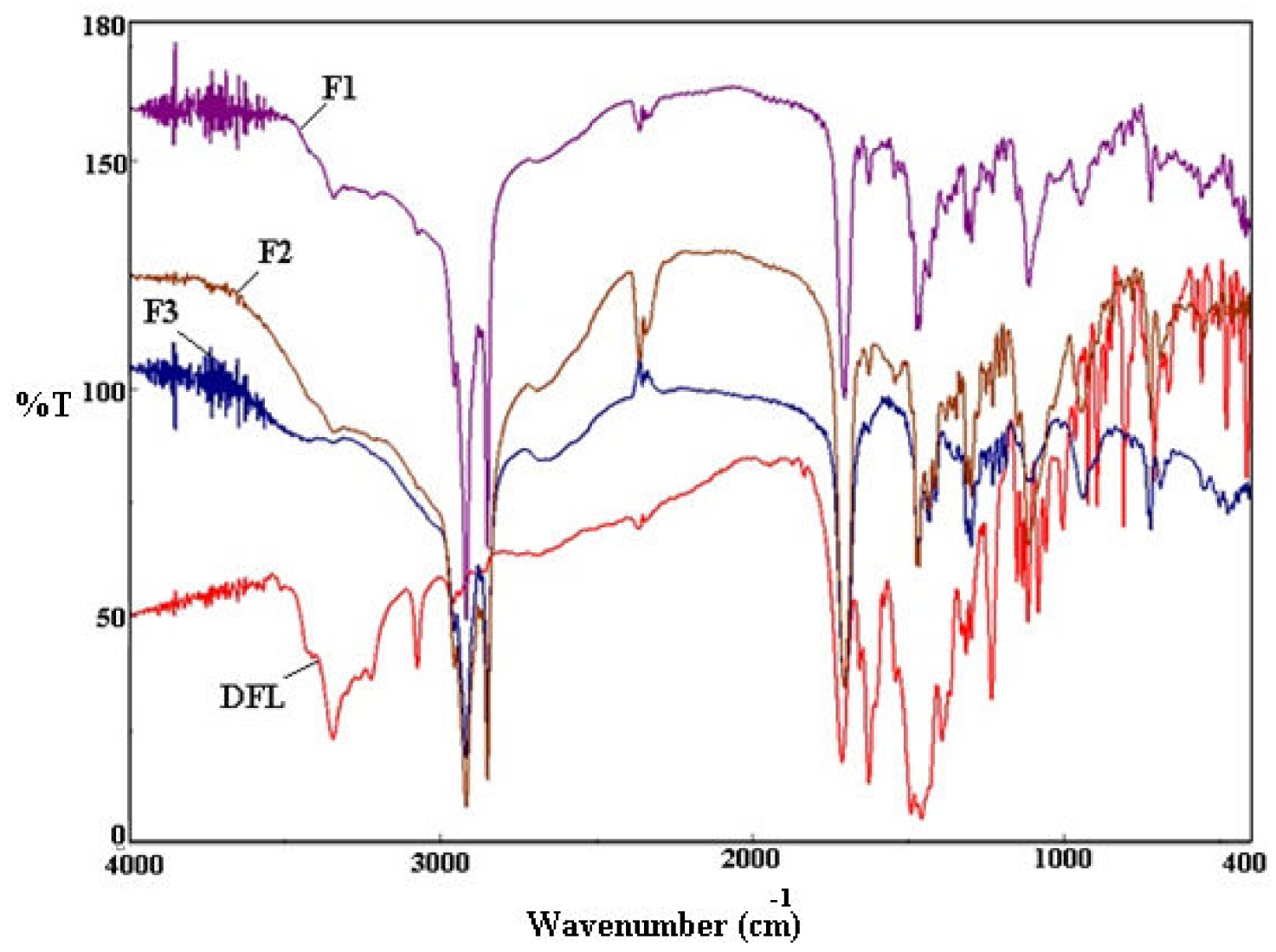

Figure 2. A comparative Fourier transform infrared (FTIR) spectra of pure DFL and DFL-loaded L-P-NPs (F1-F3).

\subsection{In-Vitro Release Studies}

In-vitro release studies were performed to know the pattern and mechanism of DFL from lipid polymer hybrid nanoparticles. A biphasic release pattern was observed from DFL-loaded L-P-NPs (F3), as shown in Figure 3. In the first phase, a burst release of the drug was observed at $4 \mathrm{~h}(81.4 \%)$, probably due to the surface-adsorbed drug and easy diffusion of the media into the nanoparticles. A sustained release of the drug was observed after $4 \mathrm{~h}$ of the study until $48 \mathrm{~h}$ in the second phase; it may be due to the lipid and polymer of the NPs, and subsequent immobilization may contribute to the slow release of the drug. The sustained release of the drug ultimately enhanced the bioavailability and, hence, reduced the dose and dosing frequency [38]. 


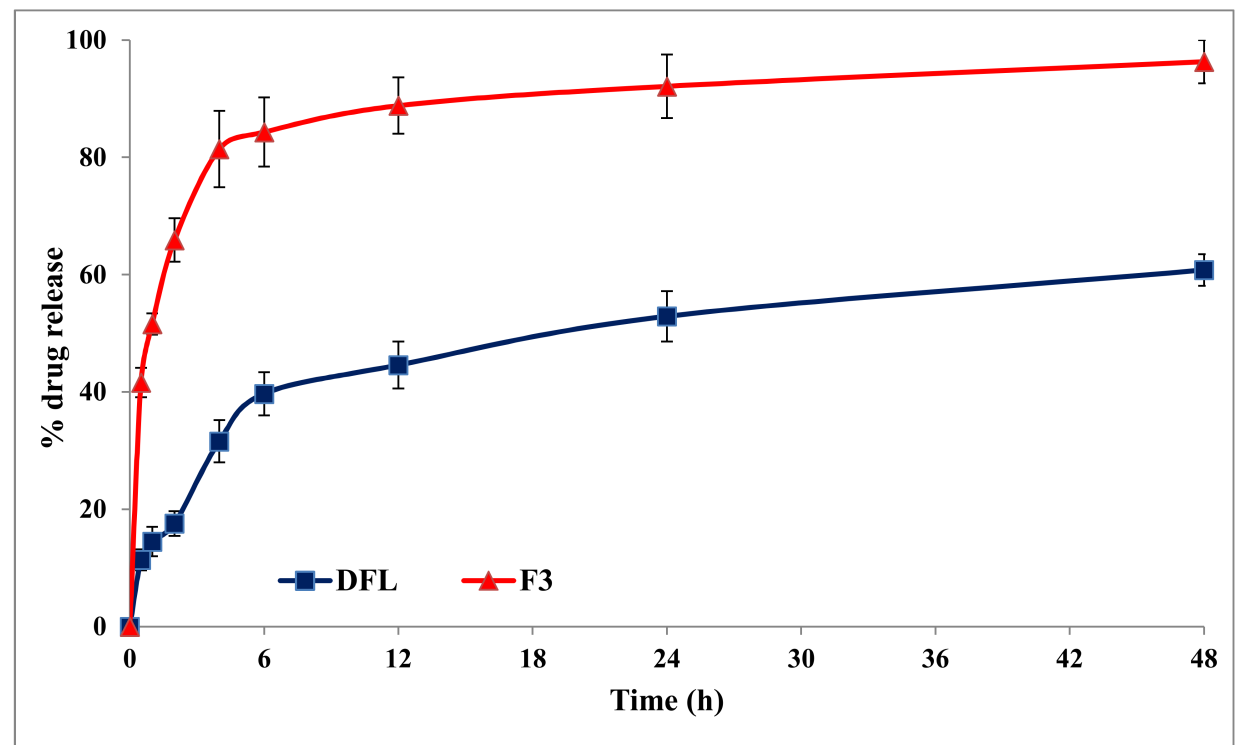

Figure 3. A comparative release profile of pure DFL and DFL-loaded L-P-NPs (F3).

\subsection{Morphology}

TEM images of the DFL-loaded L-P-NPs (F3) are shown in Figure 4. The images of the optimized formulation (F3) showed that the prepared nanoparticles were spherical in shape, having a rough surface, and no visible aggregation of the particles was observed. The size of the F3 NPs were observed approximately the same as measured by the DLS method.

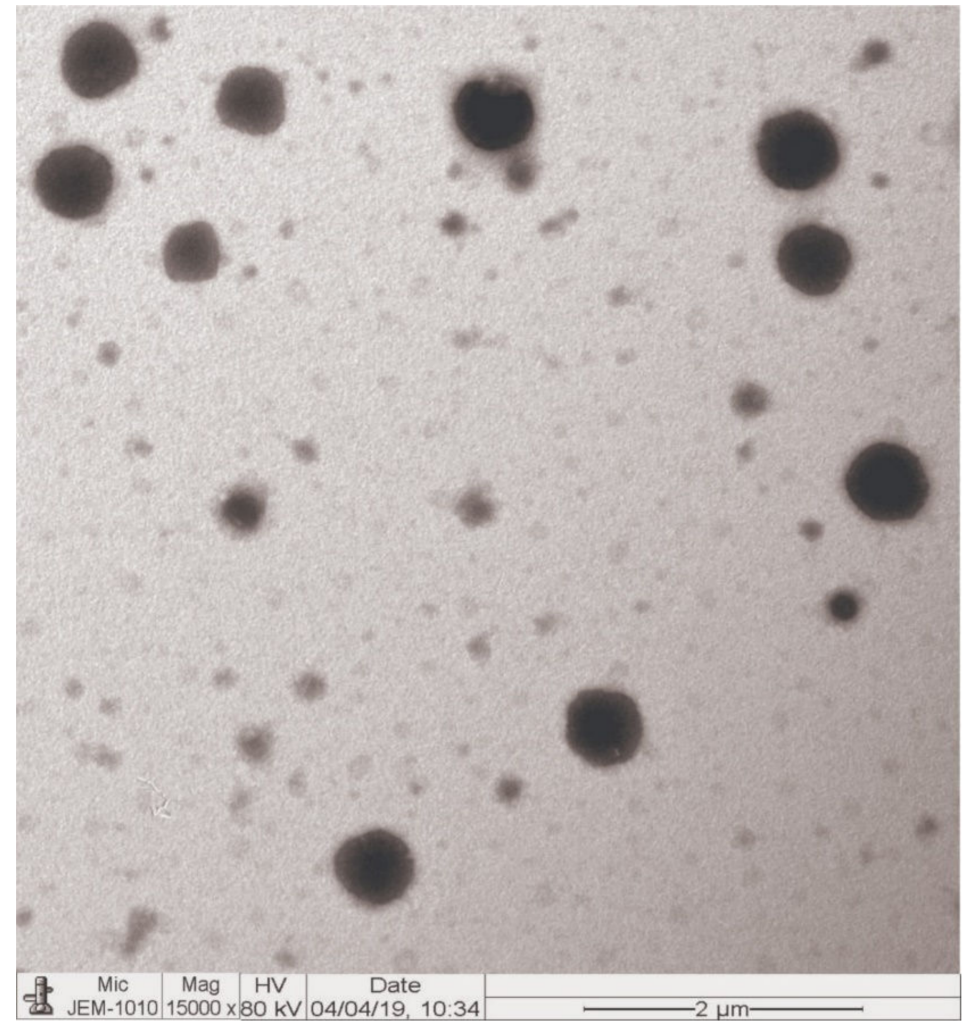

Figure 4. Transmission electron microscopy (TEM) images of optimized DFL-loaded L-P-NPs (F3). MIC: minimum inhibitory concentration. 


\subsection{In Vitro Antibacterial Activity}

Data presented in Table 3 summarizes the antibacterial activity of DFL and DFL-loaded L-P-NPs (F3) by MIC against some selected strains of Gram-positive and Gram-negative bacteria. Both activities were compared with levofloxacin as a reference standard agent. In the case of Gram-positive strains, MIC values were in the range of $0.0078-0.250 \mu \mathrm{g} / \mathrm{mL}$ and $0.0312-1.0 \mu \mathrm{g} / \mathrm{mL}$ with DFL and levofloxacin, respectively. However, the new DFL-loaded L-P-NPs (F3) demonstrated a potent activity against Gram-positive strains, with MIC values ranged between $0.0039 \mu \mathrm{g} / \mathrm{mL}$ and $0.0625 \mu \mathrm{g} / \mathrm{mL}$. These results confirmed that the MIC values of the DFL-loaded L-P-NPs (F3) appeared typically to be four-fold lower than those of DFL in the case of E. faecalis ATCC 49,532 and two-fold lower with S. epidermidis ATCC 12228. Noteworthy, DFL-loaded L-P-NPs (F3) showed MIC results with Gram-negative strains with a maximal value of $0.250 \mu \mathrm{g} / \mathrm{mL}$ in the case of $P$. mirabilis NCIMB 13,283 and a minimal value of $0.0156 \mu \mathrm{g} / \mathrm{mL}$ in the case of H. influenza ATCC 10211. Based on the MIC data, DFL-loaded L-P-NPs (F3) were two-to-four-fold more potent than those of DFL and more potent than levofloxacin by ten-fold, as shown with $P$. mirabilis NCIMB 13,283, where the MIC value decreased from $2.0 \mu \mathrm{g} / \mathrm{mL}$ to $0.250 \mu \mathrm{g} / \mathrm{mL}$. Higher potency of DFL-loaded L-P-NPs nanoparticles might be due to the unique structure of DFL with its nano-range size and large surface area.

Table 3. Minimum inhibitory concentrations (MICs) ( $\mu \mathrm{g} / \mathrm{mL})$ of DFL and its DFL-loaded L-P-NPs (F3) against bacterial pathogens compared with levofloxacin.

\begin{tabular}{cccc}
\hline Bacterial Strain & DFL & F3 & Levofloxacin \\
\hline Escherichia coil (ATCC25922) & 0.1250 & 0.0312 & 1.0 \\
Escherichia coil (ATCC 11229) & 0.1250 & 0.0625 & 1.0 \\
Escherichia coil (Clinical isolate) & 0.50 & 0.1250 & 1.5 \\
Klebsiella pneumonia (ATCC 9633) & 0.1250 & 0.0625 & 0.50 \\
Klebsiella pneumonia (Clinical isolate) & 0.0625 & 0.0312 & 0.125 \\
Pseudomonas aeruginosa (ATCC 27853) & 0.50 & 0.125 & 2.5 \\
Pseudomonas aeruginosa (ATCC 10145) & 0.50 & 0.125 & 2.0 \\
Escherichia aerogenes (ATCC13048) & 0.50 & 0.125 & 1.0 \\
Escherichia aerogenes (NCIMB 10102) & 1.0 & 0.250 & 1.5 \\
Haemophilus influenza (ATCC 10211) & 0.0312 & 0.0156 & 0.125 \\
Pseudomonas mirabilis (NCIMB13283) & 1.0 & 0.250 & 2.0 \\
Salmonella typhimurium (ATCC 14028) & 0.1250 & 0.0312 & 0.50 \\
Salmonella typhimurium (Clinical isolate) & 0.1250 & 0.0312 & 0.50 \\
Salmonella aureus (ATCC 29213) & 0.0156 & 0.0078 & 0.50 \\
Salmonella aureus (ATCC 25923) & 0.1250 & 0.0625 & 0.50 \\
Salmonella aureus (NCTC 6571) & 0.0156 & 0.0078 & 0.50 \\
Salmonella aureus (ATCC 29737) & 0.0156 & 0.0078 & 0.250 \\
Salmonella aureus (Clinical isolate) & 0.0156 & 0.0078 & 0.50 \\
Salmonella epidermidis (ATCC 12228) & 0.0078 & 0.0039 & 0.0312 \\
Salmonella pneumonia (ATCC 49619) & 0.0156 & 0.0078 & 0.50 \\
Salmonella haemolyticus (ATCC 29970) & 0.250 & 0.0625 & 1.0 \\
Bacillus subtilis (ATCC 11774) & 0.0156 & 0.0039 & 0.0312 \\
Bacillus cereus (ATCC 10876) & 0.0312 & 0.0078 & 0.0625 \\
Escherichia faecalis (ATCC 29212) & 0.0625 & 0.0312 & 0.50 \\
Escherichia faecalis (ATCC 19433) & 0.1250 & 0.0312 & 0.50 \\
Escherichia faecalis (ATCC 49532) & 0.250 & 0.0625 & 1.0 \\
\hline
\end{tabular}

\subsection{Pharmacokinetic Studies}

The comparative pharmacokinetic results obtained after an oral administration of $20 \mathrm{mg} \mathrm{kg}^{-1}$ of DFL-loaded L-P-NPs (F3) and DFL normal suspension are summarized in Table 4. As evident, both the rate and extent of absorption of DFL-loaded L-P-NPs (F3) were found to be significant $\mathrm{C}_{\text {max }}$ $(p<0.01), \mathrm{AUC}_{\text {last }}$ and $\mathrm{AUC}_{\text {tot }}(p<0.05)$ higher than the normal suspension of DFL. This results to the bioavailability of DFL-loaded L-P-NPs (F3) increased 2.3-fold in compared to the normal suspension of 
DFL, which proved the high circulation property of the nano-formulation in the circulatory system. Higher bioavailability of DFL-loaded L-P-NPs may be produced due to their ability to encapsulate the hydrophobic molecules with higher drug payloads and the improved drug stability of poorly water-soluble drugs like DFL. It has also been reported that the maintaining of the structural integrity of the nanoparticles is a critical factor for effective oral absorption of the drug to the site of action. Herein, a preparation of the hybrid form of lipid-polymer nanoparticles provides better structural integrity for nanocarriers [24]. Similar results have been reported in a previous study, where the bioavailability of enoxaparin-loaded L-P-NPs was 4.5-fold higher than an enoxaparin solution [25]. However, there are no significant changes in the half-life, elimination rate constant and mean resident time between these two formulations. These pharmacokinetic results are correlated with in vitro antibacterial activity results, where the potency of DFL-loaded L-P-NPs (F3) were found to be considerably higher than DFL against both Gram-positive and negative strains. Moreover, the results of the in vitro release profiles of DFL-loaded L-P-NPs (F3) were also comparable with our pharmacokinetic results. Overall, the bioavailability of DFL was vastly improved with the lipid-polymer hybrid nanoparticle system. The mean plasma concentration profiles of the DFL normal suspension and DFL-loaded L-P-NPs (F3) after an oral administration of $20 \mathrm{mg} \mathrm{kg}^{-1}$ are shown in Figure 5.

Table 4. Comparative pharmacokinetic profile of DFL-loaded L-P-NPs (F3) vs. normal suspension.

\begin{tabular}{ccc}
\hline Parameters & $\begin{array}{c}\text { Normal Suspension } \\
\text { (Mean } \pm \mathbf{S D}, \boldsymbol{n = 6 )}\end{array}$ & $\begin{array}{c}\text { DFL-Loaded L-P-NPs (F3) } \\
\text { (Mean } \pm \mathbf{S D}, \boldsymbol{n = 6 )}\end{array}$ \\
\hline$C_{\max }(n g / m L)$ & $231 \pm 67$ & $597 \pm 228^{* *}$ \\
$T_{\max }(h)$ & 1 & 2 \\
$A U C_{\text {last }}(n g / h / m L)$ & $1618 \pm 301$ & $3717 \pm 1600^{*}$ \\
$A U C_{\text {tot }}(n g / h / m L)$ & $2084 \pm 106$ & $3895 \pm 1508^{*}$ \\
$K_{e l}(h)$ & $0.087 \pm 0.038$ & $0.130 \pm 0.038$ \\
$T_{1 / 2}(h)$ & $6.17 \pm 0.27$ & $5.70 \pm 1.94$ \\
$M R T(h)$ & $9.72 \pm 2.04$ & $7.77 \pm 2.81$ \\
Relative Bioavailability $(\%)$ & 100 & $230 \%$ \\
\hline
\end{tabular}

$\mathrm{C}_{\max }=$ maximum plasma concentration; $\mathrm{T}_{\max }=$ Time to $\mathrm{C}_{\max } ; \mathrm{AUC}=$ Area under curve; $\mathrm{K}_{\mathrm{el}}=$ elimination rate constant; $\mathrm{T}_{1 / 2}=$ half-life; $\mathrm{MRT}=$ mean residence time. ${ }^{*} p<0.05$ significant compared with normal suspension and ** $p<0.01$ highly significant compared with normal suspension.

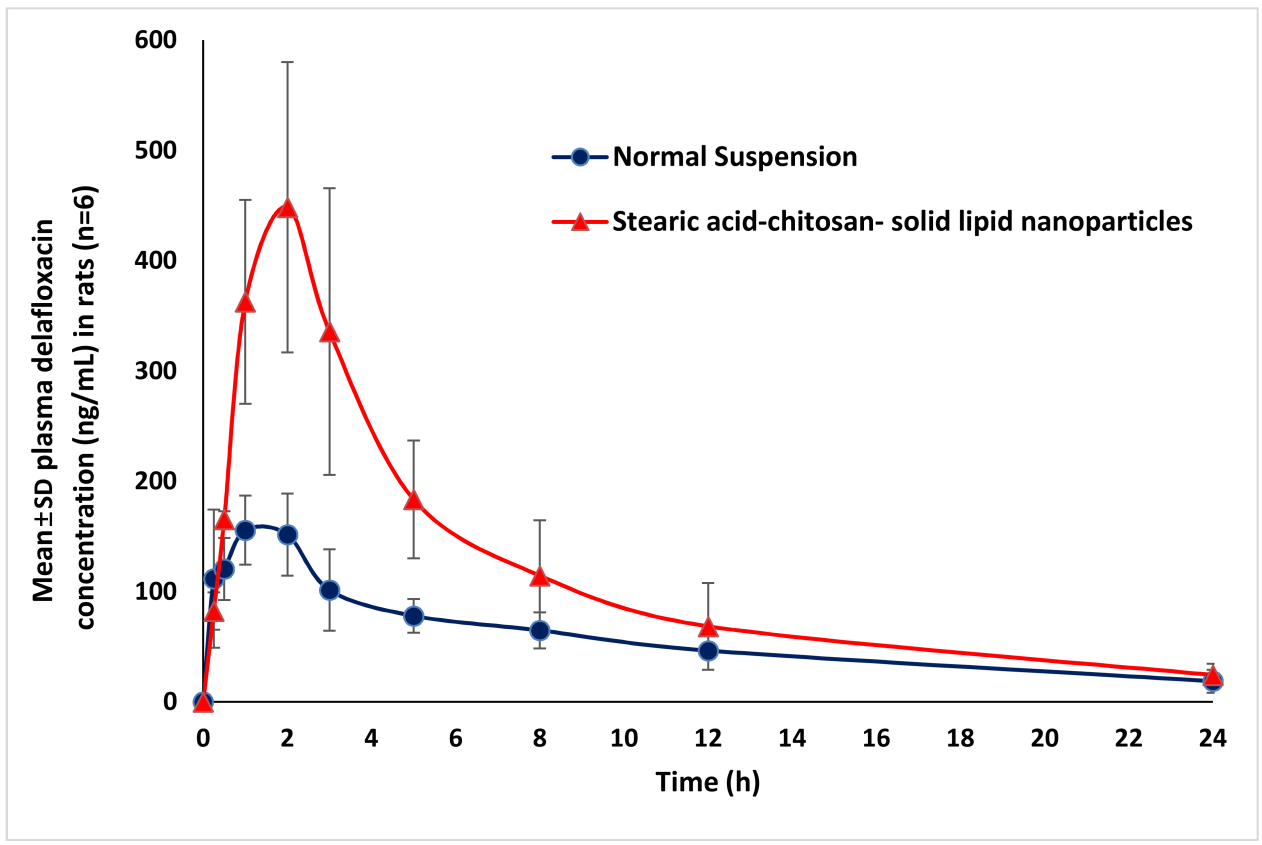

Figure 5. Comparative pharmacokinetic profile of DFL normal suspension and DFL loaded L-P-NPs (F3). 


\section{Conclusions}

In this investigation, DFL-loaded L-P-NPs (F3) were formulated and optimized with the intention to enhance their bioavailability and antibacterial activity. An in vitro drug release study showed a biphasic release pattern of the optimized formulation of DFL-loaded L-P-NPs. MIC values of the DFL-loaded L-P-NPs (F3) appeared typically to be four-fold lower than those of DFL in the case of Gram-positive strains and was 2-4-fold more potent than those of DFL against Gram-negative strains. The pharmacokinetic study in rats confirms that the bioavailability of DFL-loaded L-P-NPs was significantly higher (2.3-fold) than the DFL normal suspension. Hence, it is concluded that DFL-loaded L-P-NPs promise a better therapeutic efficacy and could be a choice of replacement for the conventional formulation, with benefits of low dose requirements and better patient compliance.

Future research will involve the possibility to carry out in vitro activity for several days to confirm the stability of activity and in vivo antibacterial studies in experimental animals.

Author Contributions: Conceptualization, M.K.A. and M.I.; methodology, M.M., M.F.A. and A.A.; MIC values, M.M.M.; writing - original draft preparation M.K.A. and M.I.; bioanalysis, E.E. and M.I.; supervision, M.I. and M.K.A.; funding acquisition, M.I. and statistical analysis, F.I. All authors have read and agreed to the published version of the manuscript.

Funding: This research was funded by the Deanship of Scientific Research at King Saud University via grant number RGP-203 and APC was also the supported by the Deanship of Scientific Research.

Acknowledgments: The authors extend their appreciation to the Deanship of Scientific Research at King Saud University for funding this work through the research group no. RGP-203.

Conflicts of Interest: The authors declare no conflicts of interest.

\section{References}

1. Falcone, M.; Concia, E.; Giusti, M.; Mazzone, A.; Santini, C.; Stefani, S.; Violi, F. Acute bacterial skin and skin structure infections in internal medicine wards: Old and new drugs. Intern. Emerg. Med. 2016, 11, 637-648. [CrossRef] [PubMed]

2. Ray, G.T.; Suaya, J.A.; Baxter, R. Incidence, microbiology, and patient characteristics of skin and soft-tissue infections in a U. S. population: A retrospective population-based study. BMC Infect. Dis. 2013, 13, 252. [CrossRef] [PubMed]

3. Jain, S.; Self, W.H.; Wunderink, R.G.; Fakhran, S.; Balk, R.; Bramley, A.M.; Reed, C.; Grijalva, C.G.; Anderson, E.J.; Courtney, D.M.; et al. CDC EPIC Study Team. Community-Acquired Pneumonia Requiring Hospitalization among U. S. Adults. N. Engl. J. Med. 2015, 373, 415-427. [CrossRef] [PubMed]

4. Markham, A. Delafloxacin: First Global Approval. Drugs 2017, 77, 1481-1486. [CrossRef]

5. US Prescribing Information of BAXDELA $A^{\mathrm{TM}}$ (Delafloxacin); Melinta Therapeutics, Inc.: New Haven, CT, USA, 2017.

6. Bassetti, M.; Pecori, D.; Cojutti, P.; Righi, E.; Pea, F. Clinical and pharmacokinetic drug evaluation of delafloxacin for the treatment of acute bacterial skin and skin structure infections. Expert. Opin. Drug Metab. Toxicol. 2017, 13, 1193-1200. [CrossRef]

7. Lemaire, S.; Tulkens, P.M.; van Bambeke, F. Contrasting effects of acidic $\mathrm{pH}$ on the extracellular and intracellular activities ofthe anti-Gram-positive fluoroquinolonesmoxifloxacin and delafloxacin against Staphylococcus aureus. Antimicrob. Agents. Chemother. 2011, 55, 649-658. [CrossRef]

8. Siala, W.; Mingeot-Leclercq, M.P.; Tulkens, P.M.; Hallin, M.; Denis, O.; Van Bambeke, F. Comparison of the antibiotic activities of daptomycin, vancomycin, and the investigational fluoroquinolonedelafloxacin against biofilms from Staphylococcus aureus clinical isolates. Antimicrob. Agents. Chemother. 2014, 58, 6385-6397. [CrossRef]

9. Remy, J.M.; Tow-Keogh, C.A.; McConnell, T.S.; Dalton, J.M.; Devito, J.A. Activity of delafloxacin against methicillin-resistant Staphylococcus aureus: Resistance selection and characterization. J. Antimicrob. Chemother. 2012, 67, 2814-2820. [CrossRef]

10. Smith, H.J.; Nichol, K.A.; Hoban, D.J.; Zhanel, G.G. Dual activity of fluoroquinolones against Streptococcus pneumoniae: The facts behind the claims. J. Antimicrob. Chemother. 2002, 49, 893-895. [CrossRef] 
11. Shiu, J.; Ting, G.; Kiang, T.K. Clinical Pharmacokinetics and Pharmacodynamics of Delafloxacin. Eur. J. Drug Metab. Pharmacokinet. 2019, 44, 305-317. [CrossRef]

12. Wu, K.; Yan, Z. FDA: Center for Drug Evaluation and Research- 208610Orig1s000. 2016. Available online: https://www.accessdata.fda.gov/drugsatfdadocs/nda/2017/208610Orig1s000,208611Orig1s000ClinPharmR. pdf (accessed on 18 August 2019).

13. Cho, J.C.; Crotty, M.P.; White, B.P.; Worley, M.V. What Is Old Is New Again: Delafloxacin, a Modern Fluoroquinolone. Pharmacotherapy 2018, 38, 108-121. [CrossRef] [PubMed]

14. Hoover, R.; Hunt, T.; Benedict, M.; Paulson, S.K.; Lawrence, L.; Cammarata, S.; Sun, E. Single and Multiple Ascending-dose Studies of Oral Delafloxacin: Effects of Food, Sex, and Age. Clin. Ther. 2016, 38, 39-52. [CrossRef] [PubMed]

15. Hoover, R.; Hunt, T.; Benedict, M.; Paulson, S.K.; Lawrence, L.; Cammarata, S.; Sun, E. Safety, tolerability, and pharmacokinetic properties of intravenous delafloxacin after single and multiple doses in healthy volunteers. Clin. Ther. 2016, 38, 53-65. [CrossRef] [PubMed]

16. Kardas, P. Patient compliance with antibiotic treatment for respiratory tract infections. J. Antimicrob. Chemother. 2002, 49, 897-903. [CrossRef] [PubMed]

17. López-López, M.; Fernández-Delgado, A.; Moyá, M.L.; Blanco-Arévalo, D.; Carrera, C.; de la Haba, R.R.; Ventosa, A.; Bernal, E.; López-Cornejo, P. Optimized Preparation of Levofloxacin Loaded Polymeric Nanoparticles. Pharmaceutics 2019, 11, 57. [CrossRef]

18. Mukherjee, A.; Waters, A.K.; Kalyan, P.; Achrol, A.S.; Kesari, S.; Yenugonda, V.M. Lipid-polymer hybrid nanoparticles as a next-generation drug delivery platform: State of the art, emerging technologies, and perspectives. Int. J. Nanomed. 2019, 14, 1937-1952. [CrossRef]

19. Wang, L.; Li, L.; Sun, Y.; Ding, J.; Li, J.; Duan, X.; Li, Y.; Junyaprasert, V.B.; Mao, S. In vitro and in vivo evaluation of chitosan graft glycerylmonooleate as peroral delivery carrier of enoxaparin. Int. J. Pharm. 2014, 471, 391-399. [CrossRef]

20. Harde, H.; Das, M.; Jain, S. Solid lipid nanoparticles: An oral bioavailability enhancer vehicle. Expert Opin. Drug Deliv. 2011, 8, 1407-1424. [CrossRef]

21. Zhang, L.; Chan, J.M.; Gu, F.X.; Rhee, J.W.; Wang, A.Z.; Radovic-Moreno, A.F.; Alexis, F.; Langer, R.; Farokhzad, O.C. Self-assembled lipid-polymer hybrid nanoparticles: A robust drug delivery platform. ACS Nano 2008, 2, 1696-1702. [CrossRef]

22. Jain, A.S.; Shah, S.; Nagarsenker, M.S.; Nikam, Y.; Gude, R.P.; Steiniger, F.; Thamm, J.; Fahr, A. Lipid colloidal carriers for improvement of anticancer activity of orally delivered quercetin: Formulation, characterization and establishing in vitro-in vivo advantage. J. Biomed. Nanotechnol. 2013, 9, 1230-1240. [CrossRef]

23. Sonvico, F.; Cagnani, A.; Rossi, A.; Motta, S.; Di Bari, M.; Cavatorta, F.; Alonso, M.J.; Deriu, A.; Colombo, P. Formation of self-organized nanoparticles by lecithin/chitosan ionic interaction. Int. J. Pharm. 2006, 324, 67-73. [CrossRef] [PubMed]

24. Khan, M.M.; Madni, A.; Torchilin, V.; Filipczak, N.; Pan, J.; Tahir, N.; Shah, H. Lipid-chitosan hybrid nanoparticles for controlled delivery of cisplatin. Drug Deliv. 2019, 26, 765-772. [CrossRef] [PubMed]

25. Dong, W.; Wang, X.; Liu, C.; Zhang, X.; Zhang, X.; Chen, X.; Kou, Y.; Mao, S. Chitosan based polymer-lipid hybrid nanoparticles for oral delivery of enoxaparin. Int. J. Pharm. 2018, 547, 499-505. [CrossRef] [PubMed]

26. Cheow, W.S.; Chang, M.W.; Hadinoto, K. The roles of lipid in anti-biofilm efficacy of lipid-polymer hybrid nanoparticles encapsulating antibiotics. Colloids Surfaces A Physicochem. Eng. Asp. 2011, 389, 158-165. [CrossRef]

27. Vieira, A.C.; Chaves, L.; Pinheiro, S.; Pinto, S.; Pinheiro, M.; Lima, S.; Ferreira, D.; Sarmento, B.; Reis, S. Mucoadhesive chitosan-coated solid lipid nanoparticles for better management of tuberculosis. Int. J. Pharm. 2018, 536, 478-485. [CrossRef] [PubMed]

28. Anwer, M.K.; Mohammad, M.; Ezzeldin, E.; Fatima, F.; Alalaiwe, A.; Iqbal, M. Preparation of sustained release apremilast-loaded PLGA nanoparticles: In vitro characterization and in vivo pharmacokinetic study in rats. Int. J. Nanomed. 2019, 14, 1587-1595. [CrossRef]

29. Anwer, K.; Mohammad, M.; Iqbal, M.; Ansari, M.N.; Ezzeldin, E.; Fatima, F.; Alshahrani, S.M.; Aldawsari, M.F.; Alalaiwe, A.; Alzahrani, A.A.; et al. Sustained release and enhanced oral bioavailability of rivaroxaban by PLGA nanoparticles with no food effect. J. Thromb. Thrombolysis. 2020, 1-9. [CrossRef] 
30. Mohammed, M.; Alnafisah, M.S.; Anwer, K.; Fatima, F.; Almutairy, B.K.; Alshahrani, S.M.; Alshetaili, A.S.; Alalaiwe, A.; Fayed, M.H.; Alanazi, A.Z.; et al. Chitosan surface modified PLGA nanoparticles loaded with brigatinib for the treatment of non-small cell lung cancer. J. Polym. Engn. 2019, 39, 909-916. [CrossRef]

31. Cockerill, F.R.; Wikler, M.A.; Alder, J.; Dudley, M.N.; Eliopoulos, G.M.; Ferraro, M.J.; Hardy, D.J.; Hecht, D.W.; Hindler, J.A.; Patel, J.B.; et al. Methods for Dilution Antimicrobial Susceptibility Tests for Bacteria that Grown Aerobically; Approved Standards, 9th ed.; CLSI: Wayne, PA, USA, 2012; p. 12. ISBN 1-56238-784-7.

32. Van Bambeke, F.; Michot, J.M.; Van Eldere, J.; Tulkens, P.M. Quinolones in 2005: An update. Clin. Microbiol. Infect. 2005, 11, 256-280. [CrossRef]

33. Brook, I.; Wexler, H.M.; Goldstein, E.J. Antianaerobic antimicrobials: Spectrum and susceptibility testing. Clin. Microbiol. Rev. 2013, 26, 526-546. [CrossRef]

34. Wiegand, I.; Hilpert, K.; Hancock, R.E.W. Agar and broth dilution methods to determine the minimal inhibitory concentration (MIC) of antimicrobial substances. Nat. Protoc. 2008, 3, 163-175. [CrossRef] [PubMed]

35. Clinical and Laboratory Standards Institute. Performance Standards for Antimicrobial Susceptibility Testing; Sixteenth Informational Supplement, CLSI Document M100-S16CLSI; CLSI: Wayne, PA, USA, 2006.

36. Iqbal, M.; Ezzeldin, E.; Herqash, R.N.; Anwer, M.K.; Azam, F. Development and validation of a novel UPLC-MS/MS method for quantification of delafloxacin in plasma and aqueous humour for pharmacokinetic analyses. J. Chromatogr. B Analyt. Technol. Biomed. Life Sci. 2020, 1138, 121961. [CrossRef] [PubMed]

37. Hanselmann, R.; Reeve, M.M. Crystalline Forms of d-Glucitol, 1-Deoxy-1-(Methylamino)-1-(6-Amino 3,5Difluoropyridine-2-yl)-8-Chloro 6-Fluoro-1,4-Dihydro-7-(3-hy Droxyazetidin-1-yl)-4-oxo-3-Quinolinecarboxylate. U.S. Patent US2016/0046603A1, 18 February 2016.

38. Anwer, M.K.; Al-Shdefat, R.; Ezzeldin, E.; Alshahrani, S.M.; Alshetaili, A.S.; Iqbal, M. Preparation, Evaluation and Bioavailability Studies of Eudragit Coated PLGA Nanoparticles for Sustained Release of Eluxadoline for the Treatment of Irritable Bowel Syndrome. Front. Pharmacol. 2017, 8, 844. [CrossRef] [PubMed]

(C) 2020 by the authors. Licensee MDPI, Basel, Switzerland. This article is an open access article distributed under the terms and conditions of the Creative Commons Attribution (CC BY) license (http://creativecommons.org/licenses/by/4.0/). 